Linguistique, littérature, didactique

\title{
Créer une métaphore en classe de $5^{\mathrm{e}}$
}

\section{Christine Duminy-Sauzeau}

\section{(2) OpenEdition}

\section{Journals}

Édition électronique

URL : https://journals.openedition.org/pratiques/2577

DOI : $10.4000 /$ pratiques. 2577

ISSN : 2425-2042

\section{Éditeur}

Centre de recherche sur les médiations (CREM)

Référence électronique

Christine Duminy-Sauzeau, « Créer une métaphore en classe de 5e », Pratiques [En ligne], 165-166 | 2015, mis en ligne le 01 octobre 2015, consulté le 08 décembre 2022. URL : http://

journals.openedition.org/pratiques/2577 ; DOI : https://doi.org/10.4000/pratiques.2577

Ce document a été généré automatiquement le 29 septembre 2020.

Tous droits réservés 


\title{
Créer une métaphore en classe de $5^{\mathrm{e}}$
}

\author{
Christine Duminy-Sauzeau
}

\section{Introduction}

1 Cet article se propose d'apporter un éclairage sur la métaphore, l'image littéraire la plus créative, à travers son apprentissage en atelier d'écriture au sein d'une séquence didactique. Après avoir posé les bases théoriques essentielles du dispositif que constitue l'atelier d'écriture littéraire, on donnera les grandes lignes d'une séquence expérimentale portant sur cette figure du discours, menée en parallèle dans quatre classes de cinquième de collèges en Rhône-Alpes. Centrer le regard sur les points nodaux lors de l'apprentissage, en suivant le processus d'écriture, ses traces écrites et les interactions langagières qui l'accompagnent, permettra sans doute, au-delà d'une meilleure connaissance des règles d'usage du dispositif et de ses atouts, de mieux connaitre cette figure du discours et les difficultés qu'elle présente dans son appropriation.

\section{Présentation liminaire}

\section{A. L'atelier d'écriture littéraire : bases théoriques majeures}

2 L'atelier d'écriture littéraire est un dispositif dont j'ai pu montrer l'efficacité au service de la didactique de l'écriture (Duminy-Sauzeau, 2010). Me plaçant dans une démarche de formation à cette animation, j'ai cherché une façon d'y former à la fois de façon plus accessible et plus efficace à long terme auprès de la didactique professionnelle. Le principal enseignement que j'en ai tiré concerne son préalable à toute action de formation, à savoir l'analyse de la structure conceptuelle de la tâche à accomplir, à partir de l'activité d'un expert. Ce préalable opératoire permet d'identifier le ou les gestes professionnels (ou concepts pragmatiques) indispensables au bon accomplissement de cette tâche, qui seront alors travaillés en formation. C'est ainsi que j'ai été amenée à modéliser le dispositif. De cette modélisation, dont le schéma figure en annexes, il apparait que 
l'atelier d'écriture littéraire est reconnaissable à la coprésence de trois caractéristiques majeures (ibid. : 335), à savoir :

- coaction entre les membres, résultat d'une conjonction ${ }^{1}$ réussie et maintenue grâce à un équilibre entre guidance et dévolution. La coaction se traduit non seulement par le fait qu'animateur et membres de l'atelier lisent et écrivent en même temps, mais par le fait qu'ils lisent et écrivent ensemble, et qu'ainsi l'avancée de l'atelier d'écriture est le résultat de l'action conjointe de tous. La mise en situation d'atelier d'écriture fait alterner les moments de guidance (puisque c'est d'abord par imitation ${ }^{2}$ que vont s'approprier les gestes à accomplir) et les moments de dévolution (ce qui désigne le fait, pour l'animateur, de déléguer une partie de son rôle);

- cohérescence au niveau des textes lus et écrits, preuve d'une intention (Bruner, 1987) dirigée vers la littérarité des textes, et obtenue grâce à un équilibre entre scrutation et déploiement. La lecture des textes, qu'il s'agisse des textes-sources qui vont aider à écrire, ou des textescibles produits au cours de l'atelier, doit se faire de façon ascendante, c'est-à-dire en partant des régularités perçues pour arriver à la structure globale qu'elles contribuent à révéler (leur "opération d'écriture majeure»). Ainsi les compétences de l'accompagnateur devront être à la fois de l'ordre de la scrutation, en quête de ces régularités, et de l'ordre du déploiement - dans une position distanciée - afin d'aider à faire apparaitre l'architecture générale du texte envisagé, à partir de laquelle on va dégager une consigne d'écriture ou de réécriture ;

- équilibre dialectique entre les éléments mis en tension dans le dispositif (langue et littérature, animateur et membre de l'atelier, projet et objet...) etc. Cet équilibre sans cesse menacé nécessite contrôle et régulation ${ }^{3}$. L'animateur qui perçoit un déséquilibre, par exemple, lors de la lecture des textes qui viennent d'être écrits, entre l'attention portée au projet d'écriture (le respect de la consigne en l'occurrence) et celle qui est portée à l'objet (le texte que l'on est en train de lire), va pouvoir proposer de choisir entre une rétroaction ${ }^{4}$ négative (afin de revenir au projet de départ) ou une rétroaction positive (afin de suivre les potentialités du texte qui s'en éloigne).

3 Ainsi l'atelier d'écriture littéraire permet-il une pratique artistique du langage, et, comme l'ont montré les études qui lui ont été consacrées (on peut citer en particulier celle de $\mathrm{J}$. Lafont-Terranova dans le cadre de sa thèse et celle de $\mathrm{C}$. Barré-de-Miniac dans le cadre de la recherche INRP qu'elle a menée avec C. Poslaniec en 2000) :

- une facilitation de l'entrée en écriture ;

- un positionnement différent de la part de l'animateur vis-à-vis des écrivants, et des écrivants vis-à-vis de l'écriture.

4 Ce type d'atelier a en outre pour caractéristique de lier lecture-écriture-étude de la langue au sein d'un projet d'écriture ${ }^{5}$.

\section{B. L'expérimentation menée : conditions et choix}

Afin de garantir la présence des trois caractéristiques majeures du dispositif ${ }^{6}$, c'est donc une séquence d'apprentissage conçue selon ce modèle qui a été proposée à quatre enseignants en classe de cinquième. Précisons que ceux-ci correspondaient à des profils contrastés du point de vue de leur formation aux ateliers d'écriture ${ }^{7}$ :

- la séquence : créée pour mes classes, elle constitue le chapitre 4 du manuel Français $6^{e}$ publié en 1994 chez Hatier (DUMINY-SAUZEAU, GRAPPIN, FAURE \& Oriol-Boyer, 1994). Pour l'expérimentation, chaque enseignant a reçu un dossier comportant tous les documents 
nécessaires à sa réalisation, du questionnaire préalable à la grille d'évaluation finale. Si le schéma général de la séquence était à suivre sur une durée de quatre heures, la répartition des séances et l'utilisation de certains documents étaient laissées à la discrétion de chacun ;

- le texte : il s'agit du « Portrait araucan en cinq touches », tiré de Vendredi ou la vie sauvage de Michel Tournier (1977) :

"C'est une mère qui te berce, c'est un cuisinier qui sale ta soupe, c'est une armée de soldats qui te retient prisonnier, c'est une grosse bête qui se fâche, hurle et trépigne quand il fait du vent, c'est une peau de serpent aux mille écailles qui miroitent au soleil. Qu'est-ce que c'est? ${ }^{8}$

- la définition de la métaphore présente dans le manuel dont la séquence émane : "Une métaphore est un rapport d'identité établi entre deux termes qui, tout en n'appartenant pas au même champ lexical, ont une qualité commune, l'un étant le comparé et l'autre le comparant. La métaphore est une image littéraire qui transforme un rapport de ressemblance en rapport d'identité » (DUMINY-SAUZEAU, GRAPPIN, ET AL., 1994 : 52) 9.

- l'opération d'écriture majeure : un procédé, la métaphore, au service d'un autre rapport au langage ;

- effet de sens : la vision du monde de Vendredi, indien de la tribu des Araucans, passe par un autre rapport au langage, imagé, qui « rapproche jusqu’à les confondre des choses parfois très éloignées les unes des autres »; il s'oppose à celui de Robinson, pour qui un papillon est un papillon et non « une marguerite qui vole»;

- fait de langue: les outils langagiers que l'on pouvait prélever dans le texte en vue d'un projet d'écriture étaient :

- le pronom, au service du genre utilisé, à savoir la devinette,

- la structure syntaxique récurrente au service du portrait en cinq touches - une pour chacune des caractéristiques,

- et la fonction attribut comme support de la métaphore ;

- la consigne d'écriture : "À la manière de Vendredi et Robinson, dans le roman de Michel Tournier, écrivez le "portrait araucan" de ce que vous voulez (être humain ou animal, phénomène naturel ou créé par l'homme, sentiment, sensation, objet, lieu, etc.). Vous l'évoquerez de façon imagée en 5 touches de plus en plus précises suivies de la question «Qu'est-ce que c'est?» de façon à ce que votre lecteur puisse deviner la réponse, mais seulement à la fin »;

- l'évaluation : la grille suivante figurait sur le même document.

Tableau 1. Grille d'évaluation

\begin{tabular}{|l|l|}
\hline Critères de réussite & Critères de réalisation \\
\hline Il s'agit bien d'une devinette & J'utilise un pronom pour remplacer le nom à deviner \\
\hline C'est un portrait & $\begin{array}{l}\text { Je pars des principales caractéristiques de ce que je } \\
\text { veux décrire }\end{array}$ \\
\hline$\ldots$ métaphorique & $\begin{array}{l}\text { Chaque touche comporte une image venant de la } \\
\text { caractéristique commune }\end{array}$ \\
\hline$\ldots$ en 5 touches & $\begin{array}{l}\text { Je reproduis } 5 \text { fois la structure : [pronom-sujet «C'«+ } \\
\text { «est »+ GN attribut] }\end{array}$ \\
\hline
\end{tabular}


Mon lecteur peut deviner, mais Je choisis l'ordre du comparant le plus lointain au plus seulement à la fin proche du comparé

6 Ainsi la séquence, conforme à la structure de base et menée à son terme, a-t-elle abouti dans tous les cas à la production, par chacun des élèves de chacune des quatre classes, d'autant de textes, que l'on a pu soumettre aux mêmes critères d'évaluation, déjà présents dans les grilles d'évaluation fournies.

7 Quelles ont été les difficultés majeures rencontrées au cours de l'apprentissage ? Une étude globale des réalisations, verbales puis écrites lors du déroulement des quatre séquences en parallèle, permettra de trouver certaines réponses.

\section{La métaphore à travers les interactions verbales}

\section{A. Représentations au regard de ses caractéristiques}

Nous poserons un premier regard transversal sur les transcriptions des quatre séquences ${ }^{10}$ afin de saisir à quels niveaux les caractéristiques de la métaphore ont pu influer sur son apprentissage. Qu'en est-il des difficultés attendues à travers les interactions langagières?

\section{La métaphore constitue un obstacle épistémologique majeur}

Pour prendre la mesure de l'obstacle majeur que représente l'approche de la métaphore par des collégiens, nous prendrons l'exemple d'une interaction langagière ${ }^{11}$ caractéristique ayant eu lieu dans l'une des classes au début de la séance 2. Lors de la lecture en commun de l'un des textes produits, le professeur recourt à un exemple :

OJ : « ... si je parle d'une une chaise< si je dis [...] c'est un objet qui a quatre pieds / ( moue dépréciative) c'est pas très intéressant parce que je suis dans le descriptif pur / moi ce que je vais essayer de faire / c'est quelque chose qui soit plus abstrait / qui parle par métaphore / c'est un animal (il soulève la chaise pour la montrer aux élèves) qui a quatre pattes (il la soulève à nouveau pour montrer les quatre pieds) c'est un animal // qui a quatre pattes"

Alexandre : « mais non mais la chaise c'est pas un animal »

OJ : « ce n'est pas un animal / c'est ça que je te dis c'est une METAPHORE ( rires des élèves et du professeur ) / C'EST ÇA QU'ON EST EN TRAIN DE FAIRE / on n'est pas dans le descriptif / ce qui nous intéresse nous c'est de comprendre comment on va faire un rapprochement avec un animal ... tout le travail consiste en la chose suivante / à faire des liens avec des choses / imaginaires des images c'est ça le principe de la métaphore / il y a une dimension poétique dans ce travail // (il repose la chaise). »

Cet extrait permet de voir l'importance de la difficulté que rencontrent les élèves au moment de produire une métaphore. L'exclamation horrifiée d'Alexandre "Mais non mais la chaise c'est pas un animal » montre bien à quel point la métaphore se présente à ceux qui la découvrent comme une transgression ${ }^{12}$ : comment peut-on faire d'une chaise, qui fait partie de son univers scolaire quotidien, un animal ? On voit dans la transcription intégrale qu'il faut toute la persuasion de l'enseignant, lors d'une longue séquence, pour venir à bout du principe de réalité de certains enfants. Difficulté à quitter la réalité, à se projeter dans l'imaginaire. Difficulté à aller dans un autre système de référence, vers l'absent, l'autre, le lointain. Difficulté aussi pour l'enseignant à conduire les élèves vers 
cela, à les accompagner pour qu'ils osent franchir le cap. Pour cela il faut du temps, mais aussi une approche scientifiquement valide.

\section{La métaphore est et semble difficile}

Dans le Gradus, l'article «Métaphore» commence ainsi : "C'est le plus élaboré des tropes ». Au-delà de l'obstacle qu'elle représente pour certains, sa reconnaissance comme sa production a semblé difficile aux élèves ainsi qu'aux professeurs, quelle que soit leur formation aux ateliers d'écriture; le mot «difficile» est répété nombre de fois et les interactions font état de ces difficultés :

OJ : « mais alors c'est un peu ... c'est compliqué cette histoire là »; et plus tard son

élève Alan : «j'ai pas trouvé de métaphores y faut que je change tout ... c'est nul »

MB, se mettant théâtralement en position d'élève: «Les métaphores, comment je vais

les trouver, ça va pas être évident... » (Duminy-Sauzeau, 2010, t.1 : 778) et, plus tard

«ce n'est pas si évident que ça de trouver des caractéristiques et de trouver la

métaphore qui correspond...»

Joris : «c'est dur à trouver Madame»

SR : « oui, c'est dur »

DL : « ...ce qui a été dur un petit peu pour vous quand vous avez écrit ça a été de

créer ça / c'est normal c'est dur » (ibid., t. $3: 1107$ ).

L'enjeu est pourtant souvent compris, même par les élèves en difficulté :

Anthony : «Monsieur, on fait comment pour inventer ?» (ibid., t. 3, 1067)

Certains ont réussi parfois à en créer mais ne s'en rendent pas compte, comme le montre cet échange :

DL Prof : « est-ce que tu as utilisé des images?»

Maria $^{13}$ (ibid. : t. 3 : 786) : « non»

DL Prof : «moi je trouve qu'à des moments oui »

Maria : « moi j'y arrive pas à en faire ».

\section{La métaphore est et semble poétique}

B. Dupriez en fait «la forme la plus condensée» de l'image littéraire, elle-même considérée comme "étant au cœur du travail poétique». Et c'est bien ainsi qu'elle est considérée en lecture, à la fois par les enseignants et les élèves :

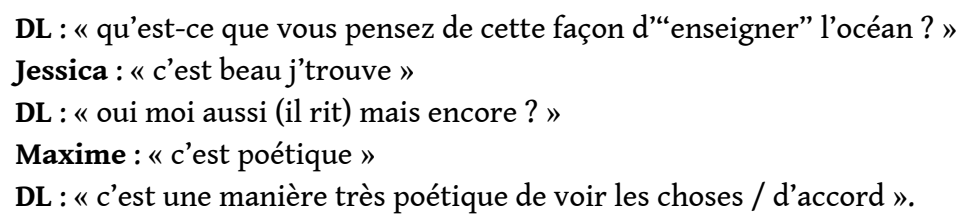

En ce qui concerne l'écriture, c'est cet « effet poétique » qui va être recherché :

OJ : « (il faut) que ça soit plus imagé ... c'est ça le principe de métaphore, c'est très imagé » (ibid., t. $3: 787,1078)$.

Souvent l'objectif semble hors de portée :

Alan : «j'suis pas du tout poétique/c'est pas du tout poétique " (il s'énerve) (ibid., t. $3:$ p. 789).

17 Cette poéticité s'est ressentie dans le choix des thèmes, spontané ou guidé par les enseignants :

$\mathrm{OJ}$ : « tu pars, d'une chose très concrète // pars d'un élément qui soit abstrait qui donne en plus des références poétiques tu as vu qu'on avait fait des textes sur la forêt c'est plus facile à partir de la forêt on avait fait plein de choses on avait fait 
des sapins par exemple l'océan c'était facile aussi c'était un univers poétique etc. essaie de trouver la même chose ... Tu comprends que si tu pars d'un élément qui est extrêmement presque trop concret et qui se prête ABSOLUMENT PAS à un univers poétique c'est plus compliqué quand même ". 96 textes, 61 concernent la nature (comme c'était le cas d'ailleurs dans le texte de Michel
Tournier), les animaux, l'amour.

\section{La métaphore est et semble personnelle} l'on tient compte de la difficulté de l'exercice - tient en grande partie à l'énergie des enseignants qui ont senti que c'était là le point nodal: les interactions langagières montrent que la création de métaphores a été travaillée jusqu'à la dernière minute des 
échanges. Rares sont les élèves qui n'en ont produit aucune. Et en examinant de près les résultats, on se rend compte que pour la plupart ${ }^{15}$ cela s'explique par la confusion avec la métonymie. On peut en conclure que, pour guider dans cet apprentissage, il faut du temps, mais aussi une approche scientifiquement valide.

\section{B. Traitement de son contenu notionnel}

\section{L'apprentissage de la métaphore nécessite un lexique de spécialité précis et complexe}

L'étude des interactions verbales montre une effervescence au niveau de la reformulation.

\section{Emploi des termes précis}

On remarque que le lexique de spécialité requis (métaphore, image, comparé, comparant, comparaison, rapport d'analogie, caractéristique) est largement utilisé par les quatre enseignants :

OJ : «Maria, est-ce que tu as utilisé des métaphores, c'est-à-dire, est-ce que tu utilisé des images pour faire une comparaison entre les choses » (ibid., t. $3: 786$ ).

Ils présentent, expliquent, réexpliquent, donnent des exemples. Mais ils font également reformuler les définitions par les élèves :

oJ : «... il s'agit d'une métaphore et ça veut dire qu'il y a deux éléments une métaphore / Eléonore, c'est quoi une métaphore? ? (ibid. , t. $3:$ :52)

Eléonore : « euh c'est un // un groupe de mots et qui vont nous faire penser à // à un autre mot ".

Le lexique de spécialité, répété en contexte, ancré dans la réalité qu'il désigne, entre en mémoire ; qu'ils soient sollicités ou qu'ils prennent la parole de façon spontanée, on voit que pour la plupart à la fois les termes et l'enjeu sont acquis :

DL : « Est-ce qu'on l'a le comparé Annaëlle?»

Annaëlle : « Non ... on n'a qu'le pronom »

DL : «oui, on n'a que le pronom parce que le comparé c'est quoi ?»

Annaëlle : « le comparé on le cherche » (ibid., t. 3bis : 1074).

Et plus tard, copiant le schéma porté au tableau, Anthony demande: "c'est quoi le comparant Monsieur, c'est "armée" ou "armée de soldats" ?"

Les métaphores produites sont identifiées par l'enseignant ${ }^{16}$, et, malgré la difficulté, par les élèves :

OJ, après la lecture d'un texte d'élève en grand groupe : « c'était bien pourquoi ? »

Elèves : «y'avait des métaphores » (ibid., t. $3:$ :778).

\section{Emploi d'« équivalents » à la validité hasardeuse}

31 La méfiance des professeurs de français concernant le lexique de spécialité, dont ils redoutent la complexité, les amène à employer des équivalents de leur cru. On remarque que chaque enseignant a ses termes de prédilection pour désigner la métaphore: métaphore, principe, image (DL : « le chien, quand tu dis "c'est un animal", c'est pas une image... », ibid. : 1130). Parfois ils en inventent : MB parle de "mot caché » pour désigner le comparé, et d'image « au ras des pâquerettes » pour désigner un cliché. Ces inventions sont heureuses. 
32 Mais il arrive que le fait de ne pas employer les termes précis engendre des confusions. Nous en donnerons deux exemples, pris dans la création des "paires oppositionnelles » qu'ils proposent entre ce qu'il faut / ne faut pas faire : - une paire oppositionnelle très employée n'a pas été bien comprise : "précis » (au sens de réel, proche du thème) / «moins parlant » ou «évasif » (au sens de plus difficile à trouver dans le cadre de la devinette) :

OJ à Maria : « tu aurais pu être plus évasive tu es restée un peu trop concrète » (ibid. : 786).

Or, s'agissant de trouver une métaphore, il était impératif, comme l'enseignant l'a d'ailleurs expliqué, d'éviter les termes « vagues » comme chose, objet, etc., d'ailleurs il avait déclaré :

OJ : « soyez précis : l'objet que j'ai choisi / quelles sont ses caractéristiques /qu'estce qui va me permettre de trouver des idées » (ibid. : 782) et s'adressant à Maryne : " au niveau du travail sur la métaphore il faut que tu trouves des choses parlantes » (ibid. : 787). - une autre confusion s'est glissée entre « points communs » et « caractéristiques ». Or, la première notion était opératoire au niveau de la lecture du portrait métaphorique de départ, pour aller du comparant au comparé, mais elle ne l'était pas au niveau de la production, puisque qu'au départ du travail seul le comparé était présent; la seconde en revanche était pertinente au niveau de leur production, puisqu'elle permettait de prendre appui sur le comparé choisi afin de créer des métaphores à partir de l'identification de chacune de ses caractéristiques :

DL : «il faut un thème, et puis trouver des points communs »

Ella, surprise : « il faut avoir un comparant pour voir les points communs »

DL, se reprenant ${ }^{17}$ « à partir de caractéristiques; ces caractéristiques peuvent nous

servir de points communs".

MB a parfaitement exposé cette différence au travers de deux schémas au tableau, démontrant à ses élèves qu'en écriture ils allaient suivre un trajet inverse de celui qu'ils avaient suivi lors de la lecture.

Par ailleurs, la proximité de la métaphore avec des notions proches permet tantôt un recours intéressant, tantôt un danger, comme nous allons le voir.

\section{Métaphore et métonymie}

C'est un danger pour le mot « métonymie » que les enseignants ont préféré à juste titre ne pas employer. Un équivalent a été trouvé dans le terme «définition»: «tu es dans la définition : il n'y a aucun moment où tu es dans la métaphore » (ibid. : 787).

Mais, il est arrivé que, faute de trouver un équivalent scientifiquement valide :

- un enseignant a employé, pour désigner le couple métaphore/métonymie, la paire oppositionnelle abstrait/concret, mais sans les apparier, l'un à une réalité absente, imaginée, l'autre à une réalité présente, celle du thème de départ. Ce qui a entraîné des confusions ;

- de même un autre enseignant a parlé dans ce cas de « description »; or, il avait appris à ses élèves que la métaphore était un outil de la description ... OJ « c'est une maison ... tu es trop dans la description ... c'est bien parce que c'est pas une maison c'est une métaphore »... «C'est une façade: tu es trop dans la description » mais en même temps il valide à juste raison en tant que métaphore ${ }^{18}$. 
On voit que les choses ne sont pas simples ...

\section{Métaphore et comparaison ${ }^{19}$}

41 La comparaison est la notion qui a été découverte rapidement au cours de la séquence, certes par erreur, mais la confusion a été levée dans les quatre classes dans la minute qui a suivi. C'est la preuve que ces deux notions sont proches : ce sont toutes deux des figures qui procèdent par analogie. Lorsqu'on a identifié l'une, on a perçu la figure et son principe fondateur : reste à percevoir la présence ou l'absence de l'outil de comparaison.

En production on peut s'appuyer sur le mot «comparaison» pour trouver une métaphore : pour qui est entré dans l'analogie, le but est proche. La question qui revient dans les interactions duelles invite à juste titre à utiliser ce tremplin : « ton thème, à quoi tu peux le comparer ? ", « à quoi tu peux penser ? ", « à quoi il ressemble?».

D’ailleurs, la comparaison est le plus court chemin pour arriver à la métaphore, et un geste qui a souvent été conseillé, voire réalisé par l'enseignant est de faire passer habilement de l'une à l'autre : un trait de crayon, et le tour est joué : (le chat) « c'est des griffes qui tranchent comme un couteau => c'est un couteau "; (la saison) « elle change comme le caméléon => c'est un caméléon » (DUMINY-SAUZEAU, $2010: 1053$ ). Reste que ce geste correctif, effectué par l'enseignant seul, aurait été plus efficace avec la complicité de l'élève concerné, qui aurait pu le reproduire, à fortiori au rétroprojecteur devant la classe entière ; c'était un moyen de trouver des métaphores à ne pas négliger.

Ainsi les enseignants ont-ils pu proposer des aides valides :

- utiliser le lexique de spécialité qui, ainsi répété en contexte, ancré dans la réalité qu'il désigne, entre en mémoire ;

- passer par la caractéristique (comme nous le verrons en analysant les traces écrites): techniquement, le recours à la caractéristique a été le plus efficace, le plus rappelé (ibid. 781-782) ;

- passer par la comparaison. Ajoutons que le passage par la comparaison rassure : Anthony, contestant qu'il soit dit de l'océan «c'est une armée de soldats", salue le développement suivant apporté par l'une de ses camarades «c'est comme si on était prisonnier» en intervenant « Oui mais Monsieur ... c'est pas une armée alors ! » après quoi Ella ${ }^{20}$ précise « on la COMPARE à une armée / c'est pas une armée » et Étienne ajoute «l'armée ça peut être les récifs qui entourent / ça fait comme une armée ";

- proposer une fiche d'évaluation (ibid. : 781) claire, nommant l'objet à produire et proposant des critères liés, comme nous allons le voir maintenant.

Malgré cela certains élèves, on l'a vu, ne sont pas parvenus à en produire. Et en moyenne pratiquement 3 essais sur 5 ne sont pas concluants après 2 temps de réécriture. Qu'est-ce qui est donc si difficile dans l'apprentissage de la métaphore? Il nous reste à identifier le chemin suivi pour créer une métaphore, à travers l'étude des brouillons.

\section{II. Étapes et points nodaux de l'apprentissage par l'étude de brouillons}

Les brouillons constituent en effet le seul accès que nous ayons à la «fabrique » des textes, et, révélant le chemin parcouru, ils permettent de repérer les obstacles et d'y remédier ainsi de façon plus rapide et surtout plus efficace. Après avoir donné quelques 
pistes permettant leur utilisation didactique, nous allons essayer de montrer comment leur étude permet de repérer les points nodaux de l'apprentissage ${ }^{21}$ en cours, ici celui de la métaphore.

\section{Préalables}

Lors de l'expérimentation, l'accès aux brouillons avait été rendu possible grâce à l'utilisation d'un support unique pour tout ce qui était écrit par les élèves au cours de l'expérimentation, mais surtout par l'apport d'une technique qui constituait, pour les élèves, une aide à la recherche des idées, à savoir le schéma heuristique proposé dans le dossier enseignant autour du mot «maison ». Ce schéma a été repris au tableau par tous les enseignants, et par la majorité des élèves - avec plus ou moins de bonheur comme nous allons le voir. La tâche à réaliser étant de faire un portrait métaphorique, cela constituait, d'un point de vue cognitif, un véritable parcours semé d'embuches. Il s'agissait en effet de partir d'un "objet » appartenant à une réalité de référence, d'en tirer 5 caractéristiques, et, à partir de chacune de ces caractéristiques, de voir à quel "objet", doté de la même caractéristique mais appartenant à une autre réalité de référence on pouvait le rapprocher, jusqu'à l'identifier. Il ne suffisait pas en effet de simplement l'en rapprocher, mais de proposer au lecteur une véritable fusion de l'un dans l'autre, une métamorphose. Il fallait ensuite justifier le choix de la métaphore en regard de l'objet, en évoquant leurs points communs. Le schéma effectué par un élève donné permet de suivre les différentes étapes de sa démarche cognitive. Nous avons fait un choix de brouillons permettant de cibler les différentes erreurs auxquelles porter remède, et les réussites à proposer en modèle, sachant que dans la mesure où elles émanent de pairs elles sont plus directement parlantes pour les élèves ${ }^{22}$. Au-delà, cela nous permet d'identifier ce qui s'avère constituer les points nodaux de l'apprentissage de la métaphore à chaque étape de son élaboration.

\section{A. Une première étape : de l'objet à la caractéristique}

\section{Difficulté à distinguer les caractéristiques entre elles}

Nombre d'élèves, appartenant pour la plupart aux groupes 3 et 4 , avaient réussi à mettre en place une schématisation minimale, mais sans parvenir à en tirer parti. C'était l'occasion de les épauler pour les aider à évoluer, sans intervenir directement sur la rédaction de leur texte ${ }^{23}$. Nous en donnons deux exemples, produits par des élèves qui ont été en grande difficulté :

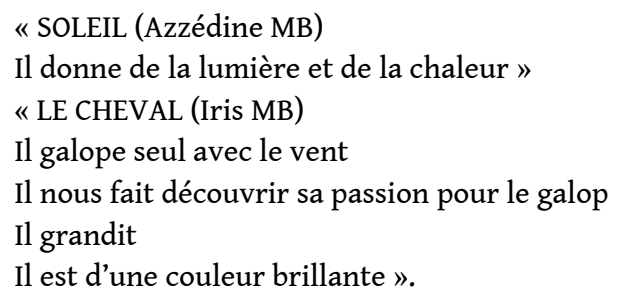

Ces deux essais présentent en quelque sorte un problème inverse. Le premier donne ensemble deux caractéristiques, le second donne une seule caractéristique (le galop) deux fois et prend pour une caractéristique du cheval ce qui est commun à tous les êtres vivants (« il grandit »). En revanche, la brillance était une caractéristique pouvant donner naissance à une métaphore. 


\section{Difficulté à prendre les caractéristiques dans la globalité de l'objet}

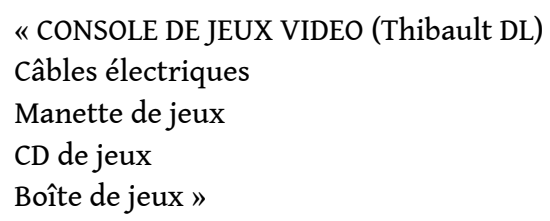

Cet essai est représentatif d'une erreur récurrente qui est de décomposer le comparé en éléments premiers, ce qui présente le danger de poser l'équivalence de la partie avec le tout. Un texte fonctionnant entièrement ainsi aurait la spécificité de substituer la métonymie à la métaphore, ce que l'on a vu dans le texte consacré à la chasse, lu par SR au début de la séance 2 et que je donne dans sa 1ère version :

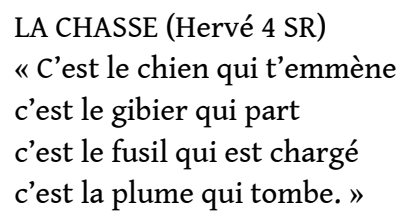

51 La meilleure preuve de la présence de métonymie, et donc de l'isotopie, est la constitution d'un champ lexical, qui est la spécificité de ce texte. La repérer permet de se donner la possibilité d'un choix, concernant ce texte, entre le projet qui l'a fait naitre (respect de la consigne qui émane du projet de l'auteur) et l'objet obtenu qui manifeste la naissance d'un autre projet, spécifique.

- revenir au projet initial suppose une rétroaction négative ;

- suivre le nouveau projet suppose une rétroaction positive respectant l'objet produit.

\section{B. Étape 2 : de la caractéristique à la métaphore}

Les trois textes suivants parviennent, même si c'est de façon très problématique, à la production d'au moins une métaphore :

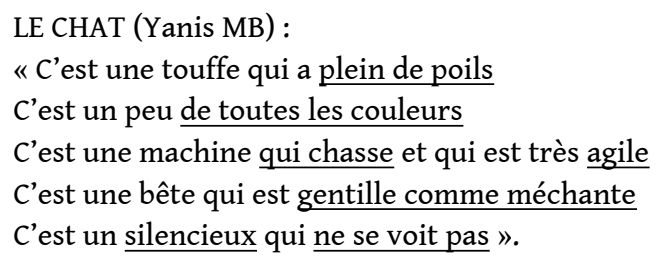

On pouvait voir que la première étape (partir d'un objet à la recherche de ses caractéristiques) avait été franchie avec succès (puisqu'on en dénombrait 7 -ici soulignées), mais qu'il semblait bien qu'au niveau de la deuxième ce qui était proposé comme métaphore était en fait à ranger au nombre des caractéristiques (exemple: "silencieux») ou des clichés (exemple: «touffe»); "bête», étant l'hyperonyme, constituait une synecdoque, et seul le mot «machine" pouvait éventuellement être considéré comme une métaphore.

LA MONTAGNE Maryne OJ :

De ce mot « montagne » placé au centre de la feuille partent cinq demi-droites conduisant chacune à un essai rédigé : il était possible de percevoir clairement à partir de ce « schéma » la distinction entre la caractéristique et la métaphore que l'on en tirait.

----- « C'est un endroit qui a de l'air pur»

----- «C'est un pic qui touche le ciel »

----- « C'est une chaîne qui est très connue » 
----- « C'est une protectrice qui garde des fleurs rares »

----- « C'est une façade sur qui on peut escalader ». dans un grand nombre de textes au niveau de la création de métaphores: en ses lieu et place on trouve des hyperonymes au contenu sémantique trop vaste («endroit») ou même des synonymes (" pic ", " chaîne »); ou encore des mots plus près de l'adjectif que $\mathrm{du}$ nom («protectrice»). Ce schéma avait l'avantage de présenter au moins une métaphore : "façade » qui, bien que très courante jusqu'à être quasiment lexicalisée, prouvait que la démarche était en cours. Ces deux textes permettent de voir que la première étape nécessaire, à laquelle le schéma permettait d'accéder, est celle qui va d'un objet à ses caractéristiques, mais que la tentation était grande, lors de la seconde, au lieu d'aller « ailleurs " pour créer des métaphores, de revenir à l'objet en en proposant des synonymes, ou des images convenues.

emarque : Il était possible aussi, à partir des deux textes qui précèdent, dont le schéma était absent ou rédigé, de proposer à la classe de retrouver le schéma qu'ils semblaient développer, afin de vérifier si leur fonctionnement correspondait bien à celui que l'on pouvait tirer du texte de l'auteur. Cette vérification permet de travailler sur un changement de registre sémiotique, dont on a montré qu'il favorisait une meilleure conceptualisation ${ }^{24}$.

LA TERRE, Seifdin DL

Nous avons sélectionné ce schéma dans lesquels est réussi le passage, même de façon minimale, du niveau 1 au niveau 2, c'est-à-dire de la caractéristique à la métaphore. Il émane d'un élève de niveau 3 (Seifdin DL), ce qui était à saluer, même si l'on peut considérer qu'il s'agit d'un cliché.

Tableau 2. Transcription diplomatique du schéma de Seifdin (au brouillon)

\begin{tabular}{|l|l|l|l|}
\hline TERRE & caractéristique & métaphore & \\
\hline touche 1 & une forme ronde & boule & qui tourne autour d'une sphère (hors schéma) \\
\hline
\end{tabular}

\section{C. Étape 3 : vers la relation entre le comparant et le comparé}

Le passage a connu des difficultés diverses comme le montrent les schémas ci-dessous :

- confusion caractéristique/métaphore (Le requin Benoît DL) :

Tableau 3. Transcription diplomatique du schéma de Benoît (au brouillon)

\begin{tabular}{|l|l|l|l|}
\hline REQUIN & caractéristique & métaphore & en fait reprise des deux précédents \\
\hline touche1 & méchant & loup & Méchant comme un loup \\
\hline touche 2 & tranchant & scie, couteau & Tranchant comme la scie ou le couteau \\
\hline touche 3 & rapide & météorite & Aussi rapide qu'une météorite \\
\hline
\end{tabular}




\begin{tabular}{|l|l|l|l|}
\hline touche 4 & attiré, aspiré & sang & Attiré et aspiré par le sang \\
\hline touche 5 & ennemi & dauphin & Ennemi du dauphin \\
\hline
\end{tabular}

- trois caractéristiques menaient bien à une métaphore et sa justification (méchant => loup => méchant comme un loup; tranchant $=>$ scie, couteau $=>$ tranchant $=>$ tranchant comme la scie ou le couteau ; rapide $=>$ météorite $=>$ aussi rapide qu'une météorite);

- "attiré » et "sang» devaient être mis sur le même plan et conduire par exemple à la métaphore du vampire; c'était le même geste correctif qui devait mettre ensemble « ennemi » et « dauphin » (ce qui est confirmé par la reprise « ennemi du dauphin »);

- la métaphore fait un détour: Le mont-Blanc (Zoé MB). Dans ce texte le comparant est affecté, non à l'objet de départ, mais à un élément de l'une de ses caractéristiques. Dans le schéma correspondant au Mont-Blanc en effet, le comparant « un enfant qui rêve » part, non du comparé Mont-Blanc, mais du mot «nuage » présent dans la caractéristique («il se perd dans les nuages»). Ce mot «nuage » ayant de plus été détourné vers son sens figuré, cela a constitué un double détour qui a perdu les lecteurs ${ }^{25}$;

- présence de toutes les étapes du parcours : Le soleil (Etienne DL). Certains élèves enfin sont parvenus sans encombre à ce dernier stade, même si ce n'est que pour deux caractéristiques, comme dans ce dernier texte. $1^{\mathrm{er}}$ niveau: les caractéristiques du comparé : «rond» « éclaire » " espace » "système solaire » (sera complété lors de la réécriture) «étoile " (ne donnera lieu à aucune métaphore). $2^{\mathrm{e}}$ niveau : les métaphores ou comparants: " gâteau " «ampoule» «cheminée» «batterie» (sera ajouté lors de la réécriture). $3^{\text {e niveau }}$ distinction comparé // comparant: "qui est brûlant » "qui éclaire plein de gens » «qui chauffe toute une planète " («qui n'a pas fini de se recharger» sera ajouté lors de la réécriture) («qui est colorée » ajouté directement à la caractéristique pour laquelle aucune métaphore n'a été trouvée).

Tableau 4. Transcription diplomatique du schéma d'Etienne (au brouillon)

\begin{tabular}{|l|l|l|l|}
\hline SOLEIL & caractéristique & métaphore & retour au comparé \\
\hline touche1 & rond & gâteau & qui est brûlant \\
\hline touche 2 & éclaire & ampoule & qui éclaire plein de gens \\
\hline touche 3 & espace ( => chauffe) & cheminée & qui chauffe toute une planète \\
\hline touche 4 & système solaire & (batterie) & (qui n'a pas fini de se recharger) \\
\hline touche 5 & & étoile & (qui est colorée) \\
\hline
\end{tabular}

\section{Remarques concernant le processus de création :}

1. "espace ", n'ayant donné lieu à aucune métaphore, a été remplacé par "chauffe " qui permettait de créer la métaphore de la cheminée ; mais il a permis de revenir au comparé « soleil » de façon judicieuse ;

2. «étoile ", placé au niveau 1 en tant que caractéristique, a été affecté d'une flèche le plaçant au niveau 2 en tant que métaphore : or, il s'agit en fait d'un hyperonyme ; on est donc dans l'isotopie, ce qui permet d'identifier une synecdoque (appelée «trope minimal»par 
B. Dupriez) et non une métaphore. D'où l'importance de la recherche de la caractéristique, à laquelle les quatre enseignants n'ont cessé d'encourager leurs élèves.

61 Montrer cette réussite, même partielle, pouvait aider l'ensemble des élèves. On le voit, les pistes ne manquaient pas autour des schémas proposés par les élèves. Ainsi l'étude des brouillons, pour y suivre le cheminement mental de l'élève, pouvait permettre de repérer les fausses routes, les chemins de traverse, que la phase de réécriture pouvait rectifier.

\section{Récapitulatif de l'aide apportée par le dispositif}

On peut, une fois analysées les difficultés rencontrées, récapituler l'aide apportée :

- mettre les élèves à l'école des écrivains, c'est-à-dire partir d'un texte d'auteur où la figure à réaliser est présente de façon massive, en prise avec un projet d'écriture lui-même porté par un fait de langue ${ }^{26}$. En effet la mise en relation avec la situation de communication entre Robinson et Vendredi, c'est-à-dire un autre rapport au langage, a porté ses fruits (DL « un portrait tel que peut le faire Vendredi et les gens de son peuple»). Se mettre à l'école des écrivains permet d'acquérir une méthode. De même le fait d'avoir à produire eux-mêmes des métaphores met les élèves, à proprement parler, « au pied du mur ${ }^{27}$;

- pratiquer la monstration et ainsi privilégier les échanges en grand groupe permettent de faire opérer la médiation entre un texte et l'autre et d'éviter un guidage excessif. L'enseignant $\mathrm{OJ}$, qui s'est mis à créer lui-même une métaphore à partir de la chaise, a poussé Alexandre dans ses retranchements. Alexandre arrivera, à partir de son thème " arbre " à produire trois métaphores: «C'est un nuage vert qui pousse, c'est un géant qui grandit d'année en année, c'est une maison qui abrite les oiseaux». Il est passé, comme ses camarades, de l'autre côté du miroir...;

- mettre les élèves en projet en suscitant une coaction qui permet à la médiation d'opérer : c'est une aide à franchir les limites ;

- permettre l'évolution de l'enseignant non formé qui, au départ, travaillait dans l'aide individuelle, la relation duelle, et a petit à petit privilégié les relations en grand groupe permettant davantage la médiation: un regard comparatif global nous a ainsi permis d'établir que l'atelier d'écriture littéraire favorisait les échanges en grand groupe, et que l'on pouvait considérer une avancée sur ce plan comme une acculturation progressive au dispositif.

\section{Conclusion}

63 La métaphore est donc ce qui échappe à la connaissance commune, ce qui va être plus personnel, surprendre. C'est cela produire une métaphore : c'est entrer dans la création, «se faire voyant». La réussite de la première métaphore évoque chez l'élève la découverte du principe alphabétique au CP : c'est de l'ordre de la révélation. C'est là la principale difficulté concernant l'apprentissage de la métaphore en production : les effets de l'apprentissage sont souterrains, jusqu'au « déclic ».

Et Michel Tournier, en grand pédagogue, le savait bien qui dans son roman prend soin d'étaler dans le temps l'apprentissage de Robinson: "Robinson commençait à comprendre. Il acceptait peu à peu que les choses les plus éloignées les unes des autres - comme la lune et un galet, les larmes et la pluie, puissent se ressembler au point d'être confondues, et que les mots volent d'une chose à une autre, même si ça devait un peu 
embrouiller les idées. Il entra tout à fait dans le jeu quand Vendredi lui expliqua les règles du Portrait araucan en cinq touches ».

Oui, on peut entrer dans le jeu quand on vous donne les règles : «Monsieur, comment on fait pour inventer?»

Les élèves ont besoin d'une aide, d'une méthode, d'un dispositif au sein duquel ils vont s'exercer sans crainte, faire des erreurs et approcher petit à petit, au cours d'un processus de production, un produit qu'ils peuvent se représenter. L'atelier d'écriture va permettre de proposer une méthode. La figure est ainsi présentée à travers ce qu'elle permet de faire : une description proposant un nouvel usage du langage. Elle est mise en relation avec l'effet de sens, à travers le contexte dans lequel elle est ancrée, et les outils langagiers nécessaires à sa production. Une aide est donnée pour sa recontextualisation dans un texte personnel.

Enfin on a pu se rendre compte qu'il n'était pas nécessaire d'avoir suivi une longue formation dans le domaine pour mettre en œuvre une séquence-modèle, et que la réalisation d'une telle séquence pouvait même constituer une formation minimale, permettant d'agir sur le geste professionnel enseignant et par conséquent sur l'apprentissage des élèves ${ }^{28}$.

68 Je dirai pour terminer qu'observer l'avancée pas à pas des élèves dans leur apprentissage de la métaphore m'a permis, grâce à l'analyse de leurs difficultés et de leurs erreurs, de m'approcher encore de cette figure de rhétorique au cœur de la poésie.

\section{BIBLIOGRAPHIE}

BARRÉ-DE-MINIAC, C. (2000) : Le Rapport à l'écriture, aspects théoriques et didactiques, Villeneuve d'Ascq, Presses universitaires du Septentrion.

BARRÉ-DE-MINIAC, C. \& POSLANIEC, C. (2000) : Le Rapport à l'écriture, aspects théoriques et didactiques, Villeneuve d'Ascq, Presses universitaires du Septentrion.

BRousseau, G. (1998) : Théorie des situations didactiques, Grenoble, Éd. La Pensée sauvage.

BRUNER, J. (1987) [1983] : Comment les enfants apprennent à parler, PARIS, RETZ.

DESNOS, R. (1995) [1944] : 30 Chantefables pour les enfants, Paris, Gründ.

DUMINY-SAUZEAU, C. (2006) : «Étude comparative de gestes professionnels enseignants : le cas de la lecture littéraire au sein d'un atelier d'écriture en classe de français », in : J.-C. Chabanne (dir.), Parler, lire, écrire dans la classe de littérature : l'activité de l'élève / le travail de l'enseignant / la place de l'œuvre, Actes des 7èmes Rencontres des chercheurs en didactique de la littérature, IUFM de Montpellier, 6-8 avril [CD-ROM].

- (2010) : L'atelier d'écriture littéraire : analyse du dispositif et de ses effets à partir d'expérimentations sur divers terrains, thèse de doctorat, Université Stendhal Grenoble 3.

- (2013), « Modéliser l'atelier d'écriture littéraire pour mieux y former », in : C. Oriol-BOYER, C. \&

D. BILous (dirs), Les Ateliers d'écriture littéraire, Paris, Hermann, p. 255-271 et 527-532. 
DUMINY-SAUZEAU, C. \& DRIOL, M. (2003), « La planification de l'écriture à partir des scénarios de Madame Bovary ", in : C. Oriol-Boyer (dir.), Critique génétique et didactique de la réécriture, Travailler avec les brouillons d'écrivains, Paris/Toulouse, B. Lacoste/CRDP Midi Pyrénées, p. 25-48.

DUMINY-SAUZEAU, C., GRAPPIN, D., FAURE, Y., ORIOL-BOYER, C. (1994) : Français $6^{\text {ème }}$, Paris, Hatier. DUMINY-SAUZEAU, C. \& TRIQUET, É. (2003) : « Mise en récit et construction des connaissances scientifiques : étude comparative de deux expériences auprès d'adultes et d'enfants ", in : M. Jaubert, M. Rebière \& J.-P. Bernié (dirs), Construction des connaissances et langage dans les disciplines d'enseignement, Bordeaux, IUFM d'Aquitaine/Université Victor Segalen Bordeaux 2 [CDROM].

DUPRIEZ, B. (1984) : Gradus. Les procédés littéraires, Paris, Union générale d'éditions.

DUVAL, R. (1995) : Semiosis et pensée humaine. Registres sémiotiques et apprentissages intellectuels, Berne, P. Lang.

FABRE, C. (1990) : Les Brouillons d'écoliers ou l'entrée dans l'écriture, Grenoble, CEDITEL/ L'atelier du texte.

GoIgoux, R. (2002), « Tâche et activité en didactique du français : contribution de la psychologie ergonomique ", in : J. Dolz, B. Schneuwly, T. Thévenaz-Christen \& M. Wirthner (dirs), Les Tâches et leurs entours en classe de français, Neuchâtel, AIRDFLM [CD-ROM].

GRESILlON, A. (1994), Éléments de critique génétique, Paris, Presses universitaires de France.

GUILLAUD, J.-C. (1998) : Enseignement et apprentissage du concept de force en classe de troisième, thèse de doctorat, Université Joseph Fourier Grenoble 1.

LAFONT-TERRANOVA, J. (2000), Pour une ethnolinguistique des ateliers d'écriture, analyse de pratiques sur plusieurs terrains, thèse de doctorat, Université François Rabelais, Tours.

MINISTÈRE DE L'ÉDUCATION NATIONALE (MEN) (1997) : Bulletin officiel de l'Éducation Nationale, 5, 30 janv.

- (2008) : Bulletin officiel de l'Éducation Nationale, numéro spécial, 28 août.

- (2014) : Conseil supérieur des programmes, 8 juin.

NUnZIATti, G. (1990) : « Pour construire un dispositif d'évaluation formatrice », Cahiers

pédagogiques, 280, p. 47-64.

ORIOL-BOYER, C. (1989) : L'Écriture du texte. Théorie, pratique, didactique, thèse de doctorat d'état, Université Stendhal Grenoble 3.

PASTRÉ, P., MAYEN, P. \& VERGNAUD, G. (2006) : « Note de synthèse : La didactique professionnelle », Revue française de pédagogie, 154, p. 145-198.

PROPP, V. (1921) : Morphologie du conte, Paris, Éd. Le Seuil, « Points ».

REY, B. (1998) [1996] : Les Compétences transversales en question, Paris, ESF Éd.

REY-DEBOVE, J. (1978) : Le Métalangage, Paris, Éd. Le Robert.

TOURNIER, M. (1977) [1971] : Vendredi ou la vie Sauvage, Paris, Gallimard.

Watzlawick, P. et al. (1972) : Une logique de la communication, Paris, Éd. Le Seuil.

WEINLAND, K., PUYGRENIER-RENAULT, J. (2000) : L'Enseignement du français au collège, Paris, BertrandLacoste. 


\section{ANNEXES}

\section{Annexes 1 : La modélisation de l'atelier d'écriture littéraire}

Le schéma ci-dessous présente la structure conceptuelle de l'atelier d'écriture littéraire, organisée selon ses trois pôles (le dispositif, ses membres et les textes qu'ils lisent et écrivent) et en suivant ses trois étapes (mise en situation, mise en jeu, accompagnement). Y figurent les concepts que nous avons dégagés comme caractéristiques de ce type d'atelier, afin de nous doter d'outils d'observation fiables pour concevoir une formation efficace.

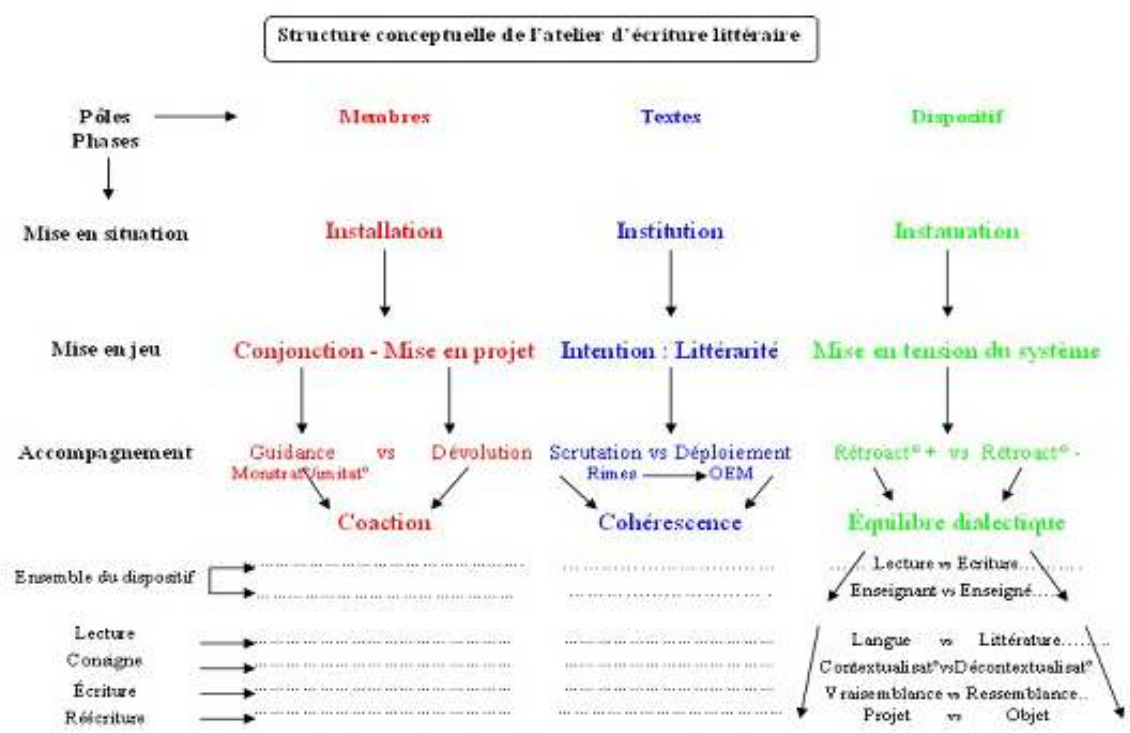

Une fois instauré le dispositif, installés les membres dans leur rôle et instituées les nouvelles règles, l'enclenchement du processus met en jeu une composante pour chaque pôle :

- la $1^{\text {re }}$, appelée "mise en tension ", concerne le dispositif qui, par son principe interne de décloisonnement, suit un mouvement dialectique entre pôles opposés terme à terme ;

- la $2^{\mathrm{e}}$, appelée conjonction, concerne les membres de l'atelier ; au-delà du lien fort que tout atelier d'écriture crée entre ses participants, elle fait en sorte qu'animateur et écrivants se sentent liés à son devenir, au sein d'un projet, né du projet de l'auteur ;

- la $3^{\mathrm{e}}$, l'intention de littérarité, désigne l'orientation de l'attention conjointe vers la littérarité des textes lus et écrits. Le « maître du jeu » qu'est devenu l'animateur doit spécifier ce qui est recherché et vers quoi on va tendre. Les contenus théoriques concernés sont la mise en œuvre de la matérialité du langage, de l'ambiguïté, des fonctions poétique et métatextuelle. Cette intention, il est nécessaire pour l'animateur de la manifester, i.e.de pratiquer la «monstration » engendrant l'imitation. Ce concept s'applique aussi bien à l'animateur de l'atelier d'écriture qu'à 
l'écrivain qui a mis en exergue la facture de son texte.

Le dispositif enclenché, il est nécessaire de procéder à son accompagnement, qui consiste dans le maintien des équilibres du système qu'il constitue, au niveau de ses 3 pôles, et cela au cours de la lecture du texte-source aussi bien que de l'écritureréécriture du texte-cible.

\section{Annexes 2 : les brouillons étudiés}

Étape 1 : De l'objet à la caractéristique : «cheval » et « console de jeux »

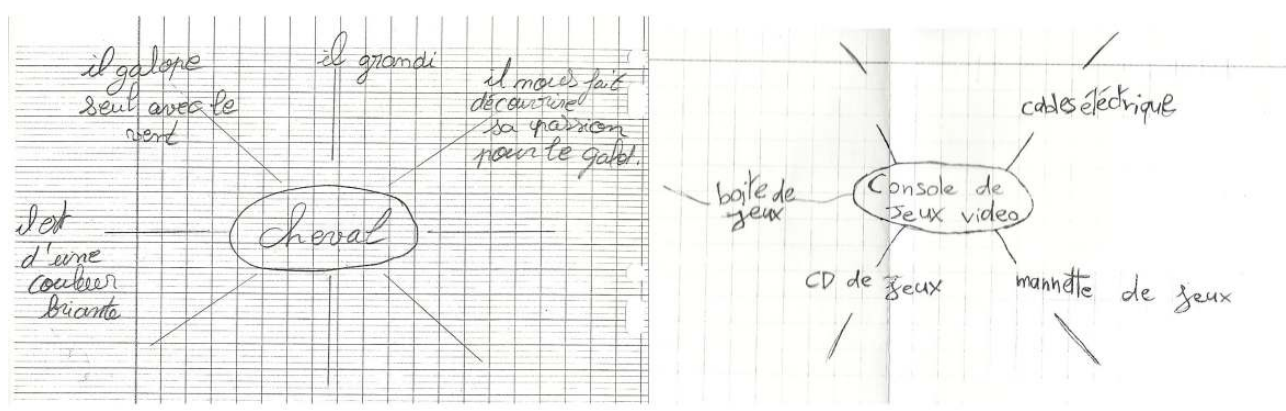

Étape 2 : De la caractéristique à la métaphore : « la terre »

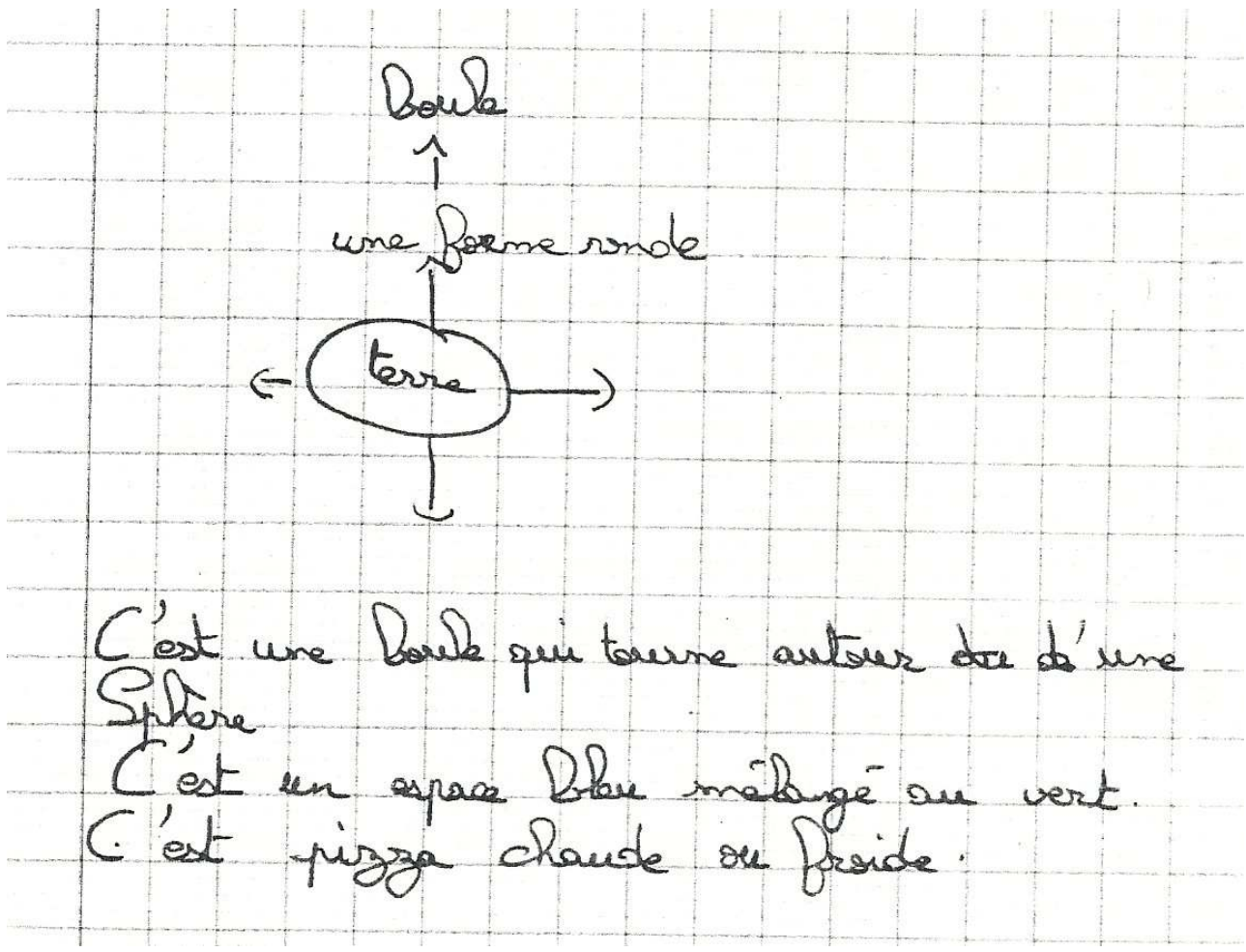




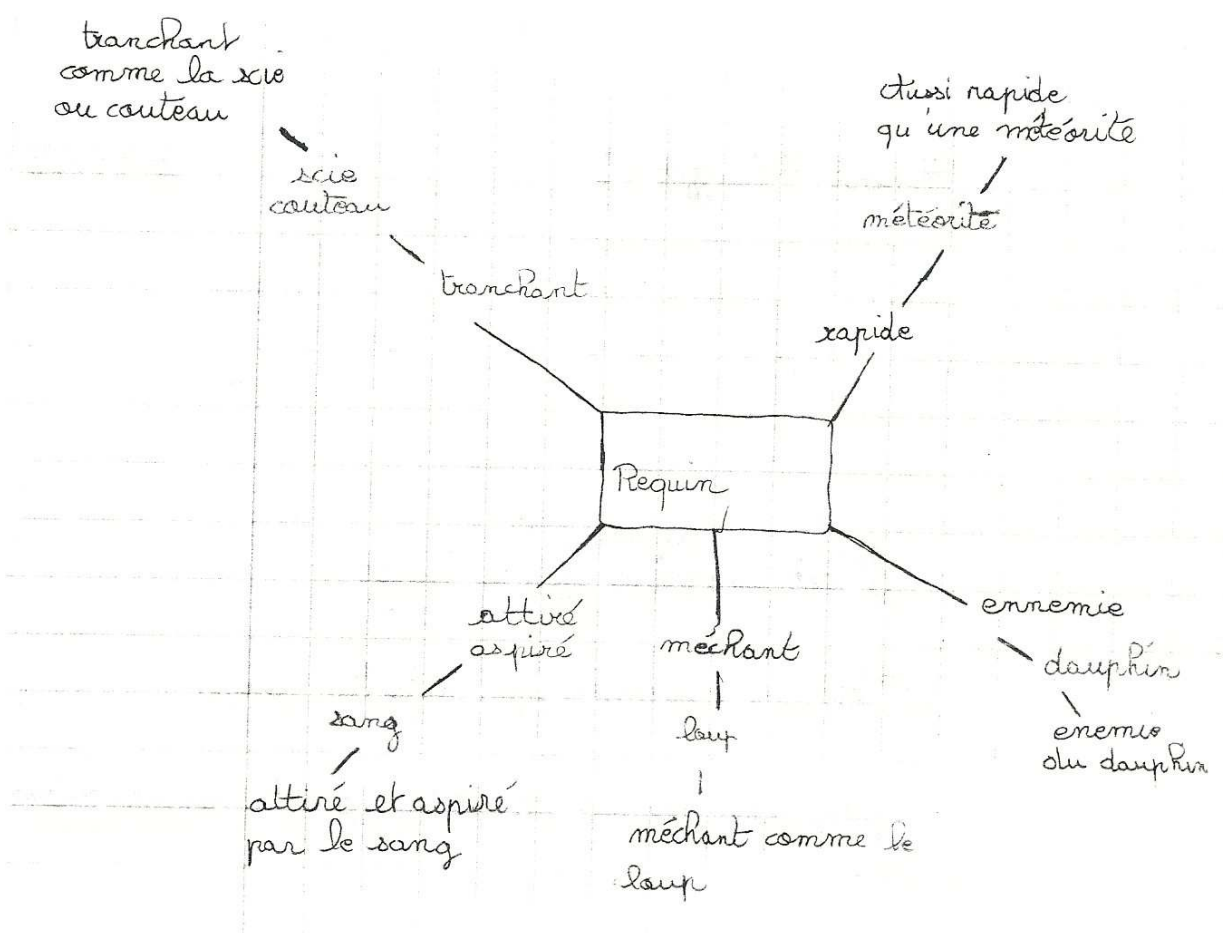

\section{NOTES}

1. À partir de la notion d' ' attention conjointe » de J. BRUNER, JEROME (1987).

2. En référence au « comme je fais » de J. Rey-Debove (1978).

3. Les termes « régulation ", " contrôle », « guidance » sont empruntés à l'évaluation formatrice. Voir G. Nunziati (1990: 48).

4. Concept emprunté à la cybernétique par les psychanalystes de l'école de Palo Alto dans leur approche systémique de la schizophrénie, que C. Oriol-Boyer a emprunté à son tour pour l'adapter à l'atelier d'écriture considéré comme un système dynamique. C'est un exemple de « concept nomade».

5. Le décloisonnement est le maitre-mot de la définition de la séquence didactique des Instructions Officielles de 1997, sous la direction de K. Weinland (MEN, 1997).

6. Rappelons qu'il s'agit, comme on peut le voir dans le schéma proposé en annexes, de la coaction de ses membres, de l'intention de littérarité vers laquelle ils tendent, et du maintien de l'équilibre au niveau de ses différentes composantes.

7. L'un d'eux, OJ, n'a reçu aucune formation spécifique; $M B$ a eu une formation «par compagnonnage » c'est-à-dire une aide « en présence » pour la mise en place d'une séquence de ce type ; SR a suivi deux stages de formation continue ; et DL a suivi les deux mêmes stages et participe depuis plusieurs années à un atelier d'écriture "de loisir » conçu selon le même modèle.

8. Pour pallier l'inconvénient qu'il y avait à couper la devinette de son contexte narratif, nous avons donné à chaque enseignant un manuel de Français $6^{e}$ de Hatier pour leur permettre la lecture des chapitres 3 et 4 intitulés respectivement «Créer une métaphore » et «Portraits » et 
retrouver ainsi à la fois l'ancrage narratif et l'analyse qui en avait été faite. Nous avons également fourni un résumé de la partie narrative.

9. Op. cit. p. 52.

10. L'ensemble des séquences menées a été filmé, et transcrit (tome 3 de la thèse, op. cit., volumes 3 et 3 bis). Le code de transcription respecte la chaîne parlée, ses débit et intonation, sans tenir compte de la ponctuation. Quand il s'agit de courts échanges, nous avons pris le parti de la rétablir. En revanche nous gardons la notation des pauses : / pour une courte pause // pour une longue.

11. Cet échange a été transcrit selon un code respectant la chaine parlée et permettant d'en signaler les caractéristiques (débit, intonation, pauses, amplitude, etc.). Nous n'avons conservé que le signalement des pauses (brèves / et longues //), l'accélération du débit (>tiens je vais...<) et l'amplitude (VAGUE).

12. On peut penser au poème de Robert Desnos (in: Chantefables pour les enfants) : «Une fourmi de 18 mètres ... ça n'existe pas!».

13. Maria est une élève du groupe 4 (c'est-à-dire en difficulté : pour évaluer dans quelle mesure les effets du dispositif étaient fonction des difficultés rencontrées par les élèves, nous les avons répartis en 4 groupes).

14. Les professeurs, conscients d'être enregistrés, suggéraient des images par gestes ou en écrivant sur la copie.

15. Un seul élève n'a produit aucune image, fût-ce par contigüité. Il s'agit d'un élève en grande difficulté pour qui l'enseignante a renégocié la tâche, lui demandant, grâce à l'emploi de synonymes cherchés à grand peine dans le dictionnaire, de ne pas répéter cinq fois le thème à l'identique ... (J. Bruner parle de «médiation de tutelle » quand un enseignant adapte la tâche dont il sent qu'elle est hors de portée de l'élève).

16. Parfois une métaphore n'est pas vue dans une production d'élève : cela vient sans doute du problème posé aux enseignants par la correction de surface qui rend difficile leur accès aux productions de leurs élèves.

17. ... alors que l'on constate encore un flottement entre comparant/comparé chez les étudiants et parfois même les professeurs en formation.

18. Ce dernier exemple est étudié dans la partie suivante consacrée aux brouillons.

19. Voir C. DUMinY-SAUZEAU (2013).

20. On remarque souvent dans la classe de cet enseignant, formé aux ateliers d'écriture, la présence d'échanges élève/élève, qui permettent de reconnaitre une situation a-didactique telle que définie par G. Brousseau (1998): la possibilité d'y voir prioritairement l'«agir» des apprenants, contrairement aux situations didactiques dans lesquelles c'est exclusivement l'«agir» de l'enseignant que l'on peut observer. C'est, avec l'importance des temps de silence, l'effet majeur de la formation à l'atelier d'écriture littéraire, que j'ai pu isoler lors de l'expérimentation, et que j'ai exposé dans ma contribution aux $7^{\mathrm{e}}$ journées des chercheurs en didactique de la littérature à Montpellier en 2006.

21. Nous avons pu développer ailleurs, de façon plus générale, la question de l'utilisation didactique du brouillon (Duminy-Sauzeau \& Driol, 2003).

22. Ajoutons que l'étude de ces brouillons aurait pu être mise en œuvre dans les classes s'il s'était agi d'une formation et non d'une expérimentation.

23. Exemple d'aller-retour entre guidance et dévolution.

24. Cf. R. Duval (1995), J.-C. Guillaud (1998) et C. Duminy-Sauzeau et É. Triquet (2003).

25. Notons que la présence de lecteurs compétents (puisqu'ayant écrit à partir de la même consigne) et bienveillants, autres que l'enseignant, est l'un des effets positifs de l'atelier d'écriture littéraire.

26. La mise ensemble d'un projet de sens et d'un fait de langue propre à lui donner toute son efficacité est l'opération d'écriture majeure. On a pu observer qu'il y a un lien direct entre la mise 
au jour de cette opération d'écriture majeure et la présence de métaphore dans le premier jet (cf. le tableau p. 476 du t. 2 de la thèse).

27. « On lit mieux ce que l'on s'est exercé à écrire » dit C. Oriol-Boyer.

28. Op.cit., T2, p. 529.

\section{RÉSUMÉS}

Après avoir posé les bases théoriques majeures du dispositif que constitue l'atelier d'écriture, et les présupposés d'une séquence didactique, on donne les grandes lignes d'une séquence expérimentale portant sur la métaphore, menée en parallèle dans quatre classes de cinquième de collège. C'est alors qu'en centrant le regard sur les points nodaux de l'apprentissage, à travers des interactions langagières et des traces écrites présentes dans les brouillons que l'on pourra, au delà d'une meilleure connaissance des règles d'usage du dispositif et de ses atouts, mieux connaître cette figure du discours et les difficultés qu'elle présente dans son appropriation.

Creation of Metaphor by Year 8 Students (Secondary 1 Students) After reminding the major theoretical bases of the device that constitutes the literary creative writing workshop and the presuppositions of a didactic sequence, I'll present the general outlines of an experimental sequence applied to the metaphor. This sequence has been studied in parallel with Year 8 students of four secondary school classes. The linguistic interactions and written traces in the drafts will focus the attention on to the nodal points of the teaching, leading, beyond a better understanding of the rules used and the strenght of the device, to a thorough knowledge of this figure of speech and the difficulties of its appropriation.

\section{INDEX}

Mots-clés : métaphore, Atelier d'écriture, Séquence didactique, Interactions langagières, Brouillons d'écoliers

Keywords : metaphor, writing workshop, didactic sequence, linguistic interactions, student's drafts 